\title{
What We Can Learn About Stick-Slip Dynamics
}

\author{
F. di Liberto ${ }^{1, a}$, E. Balzano ${ }^{1, b}$, M. Serpico ${ }^{1, c}$ and F. Peruggi ${ }^{1, d}$ \\ ${ }^{1}$ Dipartimento di Scienze Fisiche, SPIN-CNR, INFN, Università di Napoli Federico II, via Cintia, \\ I-80126 Napoli, Italy \\ adiliberto@na.infn.it, bbalzano@na.infn.it, cserpicom@gmail.com, ${ }^{\text {dp }}$ eruggi@na.infn.it
}

Keywords: friction, crossover, low- high speed.

\begin{abstract}
.
Stick-Slip motion is the basis for the description of a great variety of phenomena characterized by the presence of sliding friction between bodies with elastic features. In this article a simple experimental equipment for the analysis of this kind of dynamics is described. A wide set of possible experimental observations and measures are presented. This equipment has been tested at the university of Napoli Federico II in courses for undergraduate students and in the teacher training school for secondary education.
\end{abstract}

\section{Introduction}

A large number of natural phenomena characterized by very distinct qualitative and quantitative aspects, share common alternating dynamics in specific circumstances. These dynamics are characterized by alternating phases: static phases where the system accumulates potential energy and dynamic phases where this energy is transformed in kinetic energy. These phenomena are similar, in a more or less abstract way, to the problem of the sliding motion with friction of a body with elastic properties and are usually referred to as stick-slip processes [1-5]. Many other processes with two intermittent phases can be traced back to stick-slip processes: landslides, the motion of a windscreen wiper on a dry glass, the sound generated when a fingertip moves along the edge of a glass, the sound emission mechanism of a violin, are common examples of stick-slip processes. Many other examples may be found in a variety of mechanic processes, both artificial (braking of cars and trains) and natural (earthquake generation [10] and avalanche dynamics [9]).

The most simple physical system that exhibits a stick-slip dynamics consists of a body having mass $M$, that slides on a plane surface with friction. The body is pulled by a spring with elastic constant $k$, in such a way that the free end of the spring moves at a constant velocity $v$. For the sake of simplicity, a physical system equivalent to the former one can be considered, where the body is connected to a wall by means of a spring, while a rough plane slides at constant speed under the body. The acronym CS will be used to denote this Constrained Spring system (Fig. 1), and the acronym FS (Free Spring) to denote the previously mentioned system. The two systems are related to each other by means of a simple coordinate change.

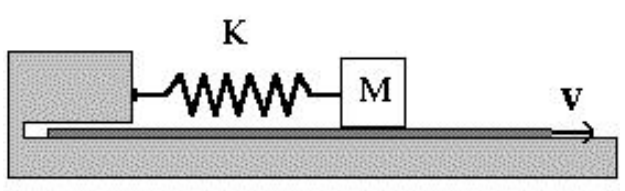

Figure 1 - Strained Spring System 


\section{Equation of motion}

Let $x=x(t)$ be the position of the body in the SS system. Assume that the origin of the reference frame is located at the position of the body at the time $t=0$, and that at $t=0$ the spring is relaxed. At $t=0$ the plane starts to move with speed $v$ under the body. The equation of motion is given by

$$
M \ddot{x}=F_{s}-F_{e l}
$$

where $F_{s}$ is the static friction and $F_{e l}$ is the elastic force generated by the spring. At each instant following $t=0$, the static friction equals the elastic force and the body remains united with the plane (stick phase). Therefore its motion is described by the equation

$$
x(t)=v t
$$

At a fixed time $t_{1}$, the elastic force of the spring

$$
F_{e l}=k x(t)=k v t
$$

equals the critical value of the static friction force

$$
F_{\max }=\mu_{s} N=\mu_{s} M g
$$

where $N=M g$ is the constraint reaction of the plane and $\mu_{s}$ is the static friction coefficient, which is assumed to be independent of the contact surface area [6,7]. Starting from $t_{1}$, the body slides back along the plane (slip phase), subject to the elastic force and dynamic friction force:

$$
F_{d}=\mu_{d} N=\mu_{d} M g
$$

where $\mu_{d}$ is the dynamic friction coefficient.

It is assumed that the dynamic friction coefficient does not depend on the speed $v$, the mass $M$, and the contact surface area [6,7]. Thus, the equation of motion for the slip phase is given by

$$
M \ddot{x}=F_{d}-k x(t)
$$

with initial conditions

$$
\left\{\begin{array}{l}
x\left(t_{1}\right)=\frac{\mu_{s} N}{k} \\
\dot{x}\left(t_{1}\right)=v
\end{array} .\right.
$$

If a new coordinate $y=y(t)$ for the position of the body is introduced

$$
y(t)=x(t)-\frac{F_{d}}{k}
$$

the equation of motion becomes

$$
M \ddot{y}=-k y(t)
$$


with initial conditions

$$
\left\{\begin{array}{l}
y\left(t_{1}\right)=\frac{\left(\mu_{s}-\mu_{d}\right) N}{k} \\
\dot{y}\left(t_{1}\right)=v
\end{array} .\right.
$$

This equation admits a solution of the type

$$
y(t)=A \cos (\omega t+\varphi)=A \cos \omega t \cos \varphi-A \sin \omega t \sin \varphi
$$

where $A$ and $\phi$ depend on the initial conditions, and $\omega^{2}=k / M$.

Chosen $t_{1}=0$ as initial time, with the initial conditions (9), the solution can be written in the form

$$
y(t)=\frac{\left(\mu_{s}-\mu_{d}\right) N}{k} \cos \omega t+\frac{v}{\omega} \sin \omega t
$$

from which $x=x(t)$ in the slip phase is given by

$$
x(t)=\frac{\mu_{d} N}{k}+\frac{\left(\mu_{s}-\mu_{d}\right) N}{k} \cos \omega t+\frac{v}{\omega} \sin \omega t .
$$

A low speed range for this equation can be considered, when the following inequality holds

$$
\frac{v}{\omega}=v \sqrt{\frac{M}{k}}<\frac{\left(\mu_{s}-\mu_{d}\right) N}{k} \text {. }
$$

In such a case the term containing sinwt in equation (12) can be neglected and the motion of the block in the slip phase is a harmonic oscillation whose amplitude $\left(\mu_{s}-\mu_{d}\right) N / k$, for fixed values of $M$ and $k$, is completely defined by the difference between static and dynamic friction coefficients (to get an idea of the orders of magnitude such that this occurs, observe that for $M=1 \mathrm{~kg}, k=1 \mathrm{~N} / \mathrm{m}$, and $\left(\mu_{s}-\mu_{d}\right) N=1 \mathrm{~N}$, equation (13) means that $v<<1 \mathrm{~m} / \mathrm{s}$ ). The slip phase actually ends after half an oscillation, when the body acquires again the speed $v$, i.e. when it is again united with the plane. Subsequently, stick and slip phases alternate periodically. A combination of the time law for the stick phase and the time law for the slip phase gives the time law plotted in Fig. 2.

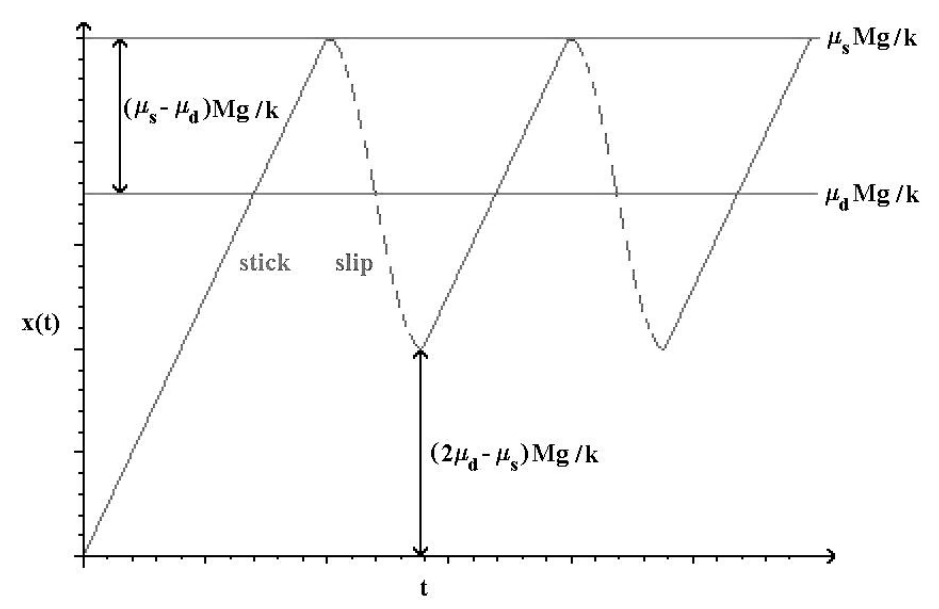

Figure 2 - Graph of $x(t)$ 


\section{Dependence of the motion on the speed of the sliding plane}

It's interesting to study the characteristic features of the motion when the speed of the sliding plane changes, forcing all the other parameters to stay constant. What happens is that, when $v$ increases, the stick phase becomes shorter and shorter, until it disappears in the infinite speed limit. Let $T_{\text {stick }}$ be the duration of the stick phase

$$
T_{\text {stick }}=\frac{2\left(\mu_{s}-\mu_{d}\right) N}{k v} \approx \frac{\text { const. }}{v} .
$$

The duration of the slip phases can be obtained differentiating relation (12) and requiring that

$$
T_{\text {slip }}=2 \pi / \omega=T
$$

This solution clearly represents the limit condition where no stick phase occurs. The other solutions of equation (8) are shown in Fig. 3 together with the values of $T_{\text {stick. }}$. For small speeds, the stick time diverges while the slip time tends to $T / 2$, i.e. just the value that has been used in the low speed approximation. When the speed increases, $T_{\text {stick }}$ exhibits a fast decrease and asymptotically tends to zero, while $T_{\text {slip }}$ tends to its asymptotic value $T$. In the neighborhood of a certain critical value $v_{\text {crit }}$ of the sliding speed (in the case shown in Figure 7, $v_{\text {crit }}=0.42 \mathrm{~m} / \mathrm{s}$ ) there is a cross-over phenomenon that quickly brings the system from configurations where $T_{\text {stick }}>>T_{\text {slip }}$ to configurations where $T_{\text {stick }}<<T_{\text {slip. }}$. For speeds much greater than this critical speed, the stick phase is practically non-existent and the motion can be approximated as a simple harmonic oscillation.

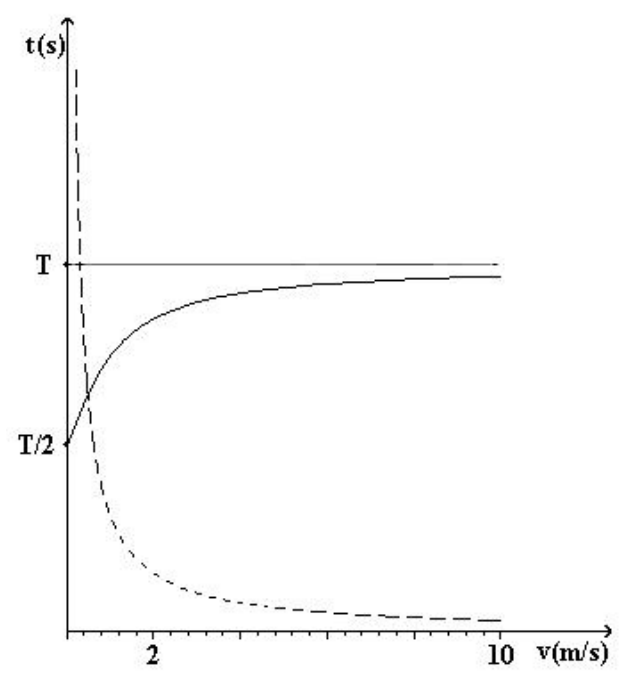

Figure 3 - Graphs of $T_{\text {stick }}$ (red) and $T_{\text {slip }}$ (blue) for the following values of the parameters: $M=1 \mathrm{~kg}, k=25$ $\mathrm{N} / \mathrm{m}, \mu_{\mathrm{s}}=1, \mu_{\mathrm{d}}=0.5$. The period $T$ of the harmonic oscillations is about $1.3 \mathrm{~s}$

\section{The experimental device}

The block is a wooden parallelepiped whose weight is approximately $650 \mathrm{~g}$ (inclusive of the weight of the position sensor mounted on top of it) with a side covered by a rubber layer. This side of the block is put on a strip of cloth whose sliding motion can be driven by means of a handle connected to a cylinder. It is not difficult to move the handle of the cylinder in such a way that the cloth slides on the workbench at an approximately constant speed. The block is connected by means of a spring to a force sensor that measures the tension of the spring. The force sensor is inserted in a flat screen, which is orthogonally locked to the wooden table around which the strip slides. On top of the block there is a position sensor (sonar) that measures the position of the block relative to the flat screen. Both the sensors are interfaced with a PC (by means of the LabPro software package). The software generates and displays directly the graphs of the observables (spring tension, position, speed, and 
acceleration of the block, and all the physical functions that can be evaluated from them) as functions of time. In Fig. 4-6 the graphs of some of the measured quantities are shown (For $M=$ $0.650 \mathrm{~kg}, k=22 \mathrm{~N} / \mathrm{M}$, and $v=0.06 \mathrm{~m} / \mathrm{s}$ ).

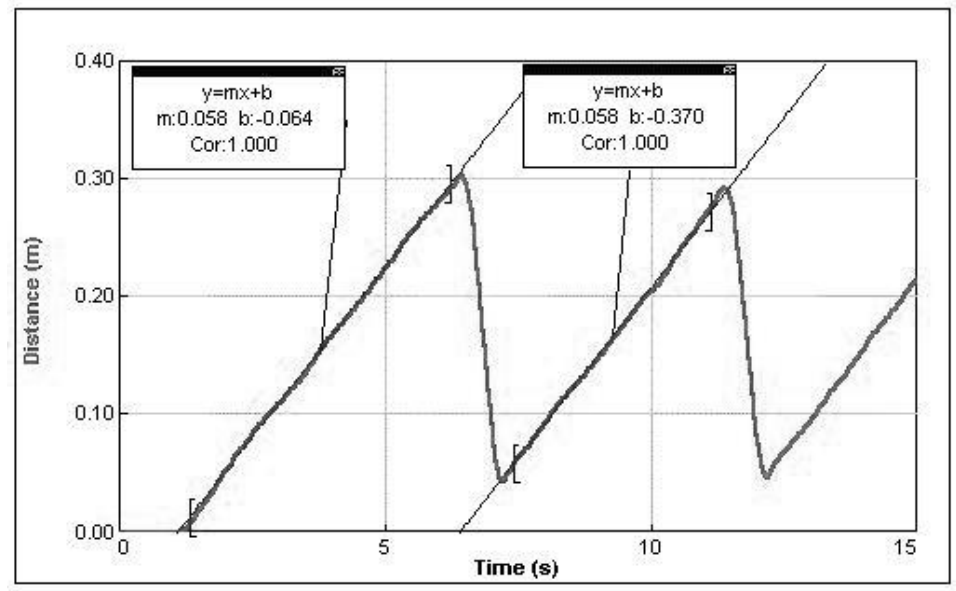

Figure 4 - Measured position of the block

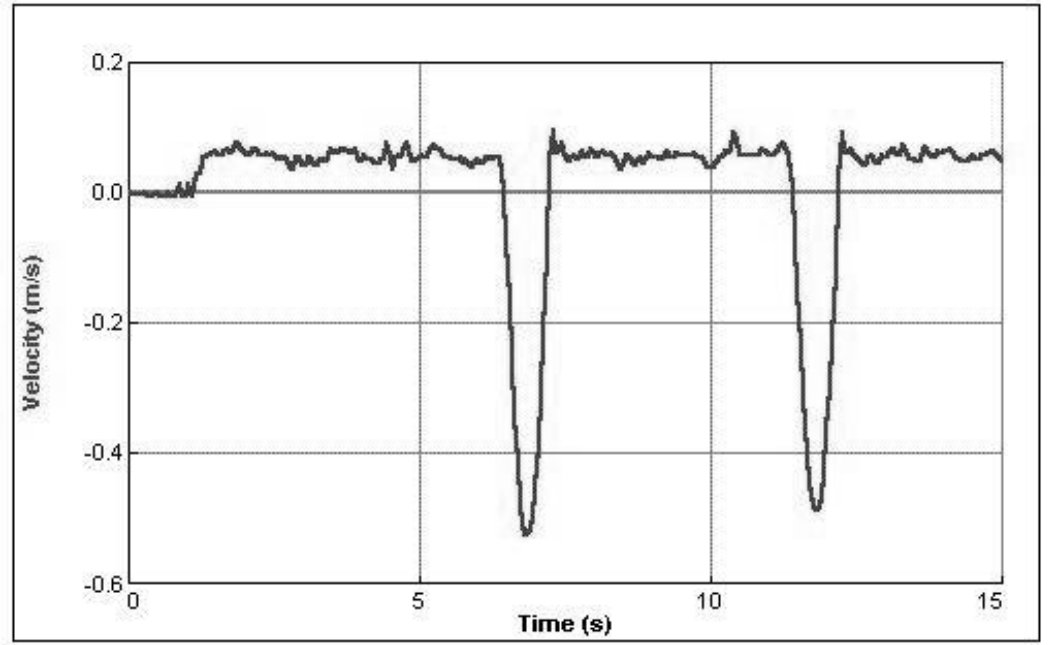

Figure 5 - Measured velocity of the block

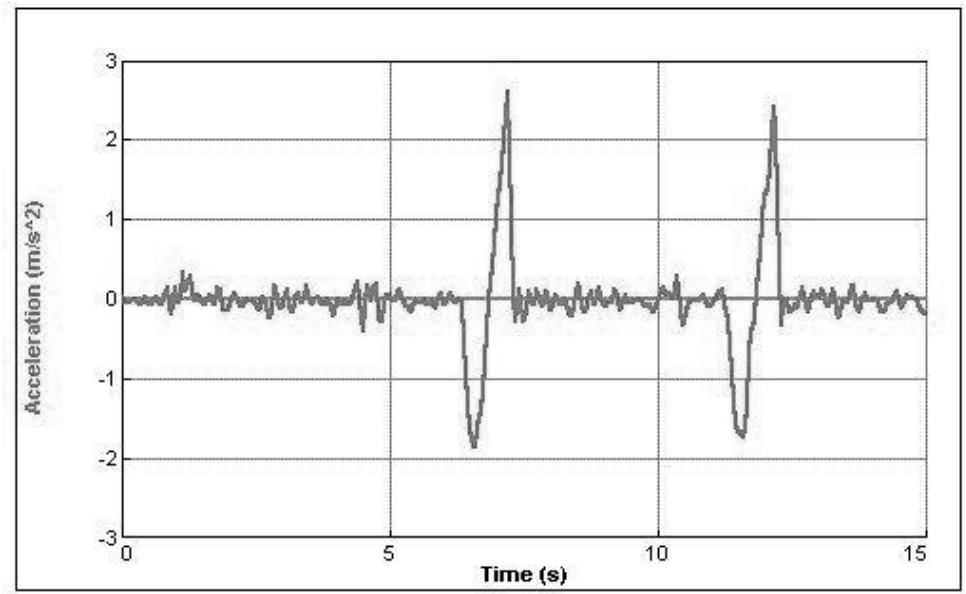

Figure 6 - Measured acceleration of the block 


\section{Conclusions}

Some of the didactic opportunities given by a simple stick-slip device designed and implemented at the University of Naples are presented in this paper. What seems to be peculiar in this experiment is that one has, on the one hand, the opportunity to perform very simple observations related to the dynamics of the block (time law, elastic force, friction) and to measure all the physical quantities related to it; on the other hand, one has the opportunity to go deeper inside the subject with more complex observations. Think of the possibility to explicit the dependence of the stick-slip dynamics on the sliding velocity or on the initial conditions, to the exploration of different types of dynamics and their connection with the model, to the possibility of displaying real-time the phase space trajectory and the mechanic energy trends. The use of computer aided sensors allows also to introduce the problems connected with transduction, sampling and numerical calculus, whose implications can also be observed real-time. During the last years, these didactic materials have been used in lab-sessions for both undergraduate students and participants to the teacher training school for secondary education at the University of Naples "Federico II".

\section{References}

[1] F.P. Bowden and D. Tabor: Friction and Lubrification (Oxford University Press 1954).

[2] E. Rabinowicz The intrinsic Variables sffecting the Stick-Slip Process Proc. Phys. Soc. 71 (1958) 668-675, and Stick and slip Sci. Am. 194 (1956),109-118.

[3] F.J. Elmer Nonlinear dynamics of dry friction J. Phys. A: Math. Gen. 30 (1997),6057-6063.

[4] B.N.J. Persson and V.L. Popov On the origin of the transition from the slip to stick Solid State Communications 114 (2000) 261-266.

[5] A.J. McMillan A non-linear model for self-exicited vibrations Journal of Sound and Vibration 205 (2000) 323-335.

[6] M. Denny Stick-slip motion : an important example of self-excited oscillation. Eur. J. Phys 25 (2004) 311-322.

[7] R. Cross Increase in friction force with sliding speed, Am. J. Phys. 73 (2005) 812-816.

[8] F.-J. Elmer The Friction Lab. http://monet.physik.unibas.ch/ elmer/flab/index.html.

[9] S.R. Nagel Instabilities in a sandpile. Rev. of Mod. Phys. 64 (1992), 32. L.A.N: Amaral and K.B. Lauritsen Energy avalanches in rice-pile model, Physica.A 231 (1996), 608-614. J. Rajchenbach Dynamic of grain avalanches, Phys.Rev.Lett. 88 (2002),14301.

[10] M.A. Moser, (1986) The response of stick-slip systems to random seismic excitation Technical Report: CaltechEERL:1986.EERL-86-03, California Institute of Technology http://caltecheerl.library.caltech.edu/167/00/8603.pdf; J. Galeano, P. Español and M.A. Rubio Experimental and theoretical results of stress relaxations in a model of earthquake dynamics, Europhys. Lett., 49 (2000) 410.

[11] L. Gratton, S. Defrancesco, A simple measurement of the sliding friction coefficient, Physics Education, vol 41, 232 (2006). 